\title{
ESTUDO PRELIMINAR SOBRE A INFLUENCIA DA ORIENTACÃO SISTEMATIZADA AO PACIENTE QUE SERÁ SUBMETIDO AO ESTUDO HEMODINÂMICO
}

\author{
Suely Itsuko Ciosak * \\ Ana Lúcia Queiroz Bezerra** \\ Hister Maria Pedroni ***
}

ReBEn/03

CIOSAK, S.I. e Colaboradoras - Estudo Preliminar sobre a Influência da Orientação Sistematizada ao Paciente que será Submetido ao Estudo Hemodinâmico. Rev. Bras. Enf.; DF, 35 : 17-38, 1982.

\section{INTRODUÇÃO}

A crescente elevação da incidência de cardiopatias adquiridas vem ocasionando o aumento de afluência de pessoas que procuram o Serviço de Hemodinâmica para elucidação diagnóstica.

Atualmente, o estudo hemodinâmico constitui um procedimento de rojtina em qualquer centro de cardiologia, visto serem suas técnicas e deduções indispensáveis para elaboração do diagnóstico preciso e para definição da conduta terapêutica a ser tomada.

No Serviço de Hemodinâmica do Hospital São Joaquim, da Real e Bene- mérita Sociedade Portuguesa de Beneficência, mensalmente, em média, 650 pacientes procuram esse serviço, sendo a maioria portadores de cardiopatias adquiridas.

Nota-se que esses pacientes, de modo geral, desconhecem o processo do estudo hemodinâmico: "como é feito, para que serve e, até mesmo, o nome do exame". Quando são informados, quase sempre o são de modo negativo. Uma das poucas informaçōes que recebe é feita através do médico, que refere "que este exame tirará dúvidas do que ele sente".

* Supervisora do Setor de Educação em Serviço da Real e Benemérita Sociedade Portuguesa de Beneficência e Docente da Escola de Enfermagem USP - São Paulo.

* Enfermeira do Serviço de Hemodinâmica da Real e Benemérita Sociedade Portuguesa de Beneficência - São Paulo.

*** Diretora do Serviço de Enfermagem da Real e Benemérita Sociedade Portuguesa de Beneficência - São Paulo. 
CIOSAK, S.I. e Colaboradoras - Estudo Preliminar sobre a Influência da Orientação Sistematizada ao Paciente que será Submetido ao Estudo Hemodinâmico. Rev. Bras. Enf.; DF, 35 : 17-38, 1982.

Tal estado de falta de informaçōes dos pacientes verifica-se pelas perguntas que freqüentemente dirigem à equipe assistencial, "é doloroso", "pode provocar a morte", "sente um calor forte, que queima o corpo itodo", "é um exame difícil", "é demorado" e "é horrível".

Todo homem tem necessidades básicas, inerentes à natureza humana, que são estados de tensōes resultantes dos desequilíbrios hemodinâmicos dos fenômenos vitais, HORTA 2, KAMIYAMA 4. A doença e a hospitalização, segundo estes mesmos autores, são fatores que determinam acentuada alteração no mecanismo de homeostase, ocasionando a manifestação de necessidades através de sinais que em enfermagem denominamse: problemas do paciente.

Constantemente recebemos pacientes emocionalmente descontrolados e tensos, o que torna difícil a realização de um exame mais rápido, com menor risco, visto ser a sua participação de suma importância para o sucesso do estudo.

As alteraçōes fisiológicas são facilmente detectadas, o que nem sempre occrre com as de ordem psicossocial; já que poucas são as informaçōes sobre métodos de atendimentos comprovadamente eficientes.

Diante do exposto, sentiu-se a necessidade de prestar uma orientação no pré-estudo hemodinâmico, que viesse diminuir a apreensão, bem como preparar os pacientes para maior colaboração durante o exame, pois é conhecida a influência da assistência psicológica no sucesso de cirurgias, como cita FONTE:S ${ }^{1}$, ou em qualquer outro procedimen'to ou tratamento durante a hospitalização, KAMIYAMA 3

Havia, porém, um problema com relação a determinaçōes de quando poderia ser feito esse tipo de assistência, já que os pacientes que iam para cirurgia estavam internados e os que se subme- teriam ao estudo hemodinâmico, na sua maioria, viriam diretamente do seu domicílio.

A partir daí foi elaborada uma orientação sistematizada aos pacientes abordando os seguintes aspectos:

$$
\begin{aligned}
& \text { - finalidade do exame } \\
& \text { - duração do exame } \\
& \text { - reações e sensaçōes sen'tidas } \\
& \text { - descrição sumária da técnica em- } \\
& \text { pregada } \\
& \text { - como poderá colaborar com o } \\
& \text { exame. }
\end{aligned}
$$

No entanto, não houve, até o momento, avaliação dos efeitos dessa orientação no desenvolvimento do exame, principalmente no aspecto emocional. Decidiu-se então elaborar uma pesquisa que visasse ao efeito da orientação que estava sendo desenvolvida ao paciente a ser submetido ao estudo hemodinâmico.

\section{OBJETIVO GERAL}

Avaliar a eficácia da orientação sistematizada no preparo psicológico do paciente submetido ao estudo hemodinâmico.

Objetivos Específicos

Verificar:

- grau de conhecimento do paciente em relação ao exame

- grau de ajuda promovida ao paciente na realização do exame

- comportamento do paciente durante a realização do exame.

\section{MATERIAL E MÉTODOS}

O trabalho foi realizado no Serviço de Hemodinâmica do Hospital da Real e Benemérita Sociedade Portuguesa de Beneficência, que possui 760 leitos, sendo que destes, $60 \%$, aproximadamente, 
CIOSAK, S.I. e Colaboradoras - Estudo Preliminar sobre a Influência da Orientação Sistematizada ao Paciente que será Submetido ao Estudo Hemodinâmico. Rev. Bras. Enf.; DF, 35 : 17-38, 1982.

se destinam a pacientes com cardiopatias, independentemente de sua situação previdenciária e econômica.

O Serviço de Hemodinâmica conta com 3 salas equipadas e aparelhadas onde diariamente são realizados de 30 a 36 exames, sendo duas equipes médicas responsáveis por este atendimento no plantão da manhã a tarde, ficando o plantão da noite para atender exames que se prolongaram no decorrer do dia e emergências.

Uma terceira equipe opera aos sábados, onde são realizados uma média de 3 exames.

Nos plantōes manhã e tarde, além de técnicos e atendentes responsáveis pelo controle e operação das máquinas filmadoras, reveladoras, 'transporte de pacientes e provisão de material estéril, 6 operadoras de sala (auxiliares de enfermagem treinadas) atuam por plantão. A noite, este número é reduzido para 3, devido aos motivos citados.

A grande maioria dos pacientes são admitidos diretamente neśte Serviço, onde aguardam em 2 salas de espera o momento em que irão realizar o estudo hemodinâmico.

A pesquisa foi realizada em 2 grupos de pacientes, um que não recebeu a orientação antes de realizar o exame (Grupo B), outro que recebeu orien'tação de modo sistemático (Grupo A).

A população de pacientes que iriam se submeter ao estudo hemodinâmico deveriam preencher os seguintes requisitos:

\footnotetext{
- ambos os sexos

- maiores de 15 anos

- lúcidos e que não iriam se submeter a anestesia geral

- sem procedência de Unidade de Terapia Intensiva ou Unidade de Emergência
}

- pertencer a uma só equipe de médicos hemodinamicistas.

Para coleta de dados foram elaborados 3 formulários, sendo que 2 foram aplicados ao grupo que iria receber a orientação (Grupo A), um antes de recebê-la e outro após o término do exame.

A fim de obter maior controle, elaborou-se $0 \quad 3 .^{\circ}$ formulário, que seria aplicado no pós-exame dos pacientes eventualmente não orien'tados, devido ao seu não comparecimento no horário estipulado para admissão, ou devida à urgência diagnóstica.

Foi estabelecido o período de 11 a 30 de maio de 1981 para efetuar a coleta de dados.

A aplicação dos formulários foi feita por 4 enfermeiras, sendo 3 elaboradoras do presente trabalho.

Para o grupo A, o formulário foi aplicado em uma das salas de espera, já descrita, de modo a se proceder à entrevista individualizada, antes de ser ministrada a orientação.

Os formulários do pós-exame foram preenchidos com o paciente já na unidade de internação.

Para que os resultados fossem mais fidedignos, foi padronizada uma orientação e executada por 2 enfermeiras integrantes do Serviço de Hemodinâmica e Setor de Educação em Serviço.

Paralelamente, desenvolveu-se um programa de reorientação às operadoras de sala, quanto ao atendimento a ser prestado ao paciente, na sala de exames, durante a realização do mesmo. Os aspectos abordados nessa orientação estão relatados no anexo.

\section{ANÁLISE, RESULTAdos E DIS- CUSSĀO}

No período proposto, foram entrevistados 63 pacientes no grupo A, sendo 
CIOSAK, S.I. e Colaboradoras - Estudo Preliminar sobre a Influência da Orientação Sistematizada ao Paciente que será Submetido ao Estudo Hemodinâmico. Rev. Bras. Enf.; DF, $35:$ 17-38, 1982.

$38(60,3 \%)$ do sexo masculino e 25 $(39,5 \%)$ do sexo feminino (tabela 1 ).

O grupo B foi constituído por 48 pacientes, dos quais $29(60,4 \%)$ pertencem ao sexo masculino e $19(39,5 \%)$ ao sexo feminino (tabela 1 ).

$\mathrm{Na}$ tabela 1, observa-se maior incidência de pacientes do sexo masculino e deduz-se ainda que o nível de escolaridade predominante é o primário, para ambos os sexos e grupos. No sexo masculino, nota-se maior grau de instrução, inclusive o universitário, que corresponde a $17,4 \%$ no grupo A e $8,3 \%$ no grupo $B$. Vê-se também que o maior indise de analfabetismo recai no grupo $B(11,6 \%)$.

Na tabela 2, vê-se que a ocupação principal para o sexo feminino é prendas domésticas, para os dois grupos, onde se encontra a incidência de $13,6 \%$ e $12,6 \%$, respectivamente. Para o sexo masculino observa-se que o maior número é de aposentados $(7,2 \%)$ para o grupo A e comerciários $(6,3 \%)$ para o grupo B. Verifica-se que a ocupação de prendas domésticas, que é pouco valorizada como fator desencadeante nas cardiopatias adquiridas é de grande evidência nos grupos estudados.

No grupo B, observa-se ainda que o número de pacientes com mão-de-obra não especializada é significativo, e corresponde a 6,3\% dos pacientes estudados. Estes dados coadunam com os resultados observados na tabela 3 e 4 , analisadas a seguir, onde a faixa etária não permite atividades diversifisadas.

Na população estudada, para o grupo A, nota-se predominância na faixa etária de 50 a 59 anos, $31,8 \%$, dos quais $22,2 \%$ são do sexo masculino e $9,5 \%$ do sexo feminino. Logo a seguir temos pacientes entre 40 a 49 anos, sendo que neste intervalo encontramos maior número de pacientes do sexo feminino $(11,1 \%)$ (tabela 3 ).

Para o grupo $\mathrm{B}$, a incidência é semelhante, predominando a faixa de 40 a 49 anos para o sexo feminino $(16,6 \%)$ e de 40 a 5.9 anos para o sexo masculino $(41,6 \%)$ (tabela 4$)$.

Observa-se nas tabelas 5 e 6 que maior número de pacientes provêm de outros Estados, perfazendo um total de $44,4 \%$ para o grupo A e $52,1 \%$ para o grupo $\mathbf{B}$. 
CIOSAK, S.I. e Colaboradoras - Estudo Preliminar sobre a Influência da Orientação Sistematizada ao Paciente que será Submetido ao Estudo Hemodinâmico. Rev. Bras. Enf.; DF, 35 17-38, 1982.

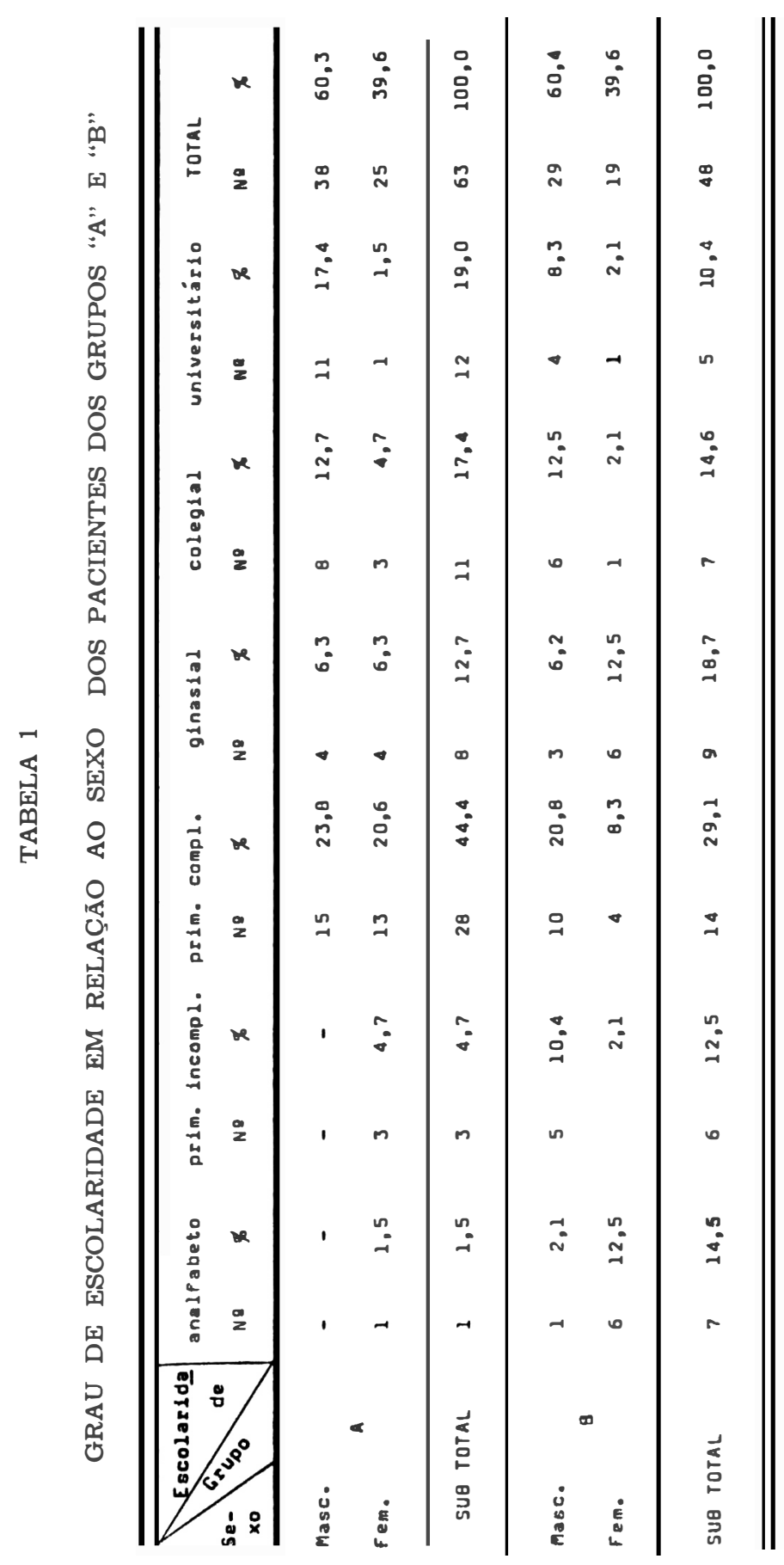


CIOSAK, S.I. e Colaboradoras - Estudo Preliminar sobre a Influência da Orientação Sistematizada ao Paciente que será Submetido ao Estudo Hemodinâmico. Rev. Bras. Enf.; DF, 35 : 17-38, 1982

TABELA 2

TIPO DE OCUPAÇÃO DE ACORDO COM O SEXO DOS PACIENTES DOS GRUPOS “A” E “B”, SUBMETIDOS AO ESTUDO HEMODINÅMICO.

\begin{tabular}{|c|c|c|c|c|c|c|c|}
\hline \multirow{2}{*}{ 0cupação } & \multirow[t]{2}{*}{ Sexo } & \multicolumn{2}{|c|}{ Masculino } & \multicolumn{2}{|c|}{ Feminino } & \multicolumn{2}{|c|}{ TOTAL } \\
\hline & & Ne & 5 & Ne & $\varnothing$ & Ne & $\%$ \\
\hline \multirow{2}{*}{ Prendas domésticas } & A & - & - & 15 & 13,5 & 15 & 13,5 \\
\hline & $\mathbf{B}$ & - & - & 14 & 12,6 & 14 & 12,6 \\
\hline \multirow[t]{2}{*}{ Rpesentado } & A & 8 & 7,2 & 3 & 2,7 & 11 & 9,9 \\
\hline & B & 1 & 0,9 & - & - & 1 & 0,9 \\
\hline \multirow{2}{*}{ Comerciério } & A. & 5 & 4,5 & - & - & 5 & $\Delta, 5$ \\
\hline & B & 7 & 6,3 & 1 & 0,9 & 8 & 7,2 \\
\hline \multirow{3}{*}{$\begin{array}{c}\text { Aduinistrador, Advoga } \\
\text { do }\end{array}$} & A & 5 & 4,5 & 2 & 1,8 & 7 & 6,3 \\
\hline & a & 3 & 2,7 & 1 & 0,9 & 4 & 3,6 \\
\hline & $A$ & 3 & 2,7 & - & - & 3 & 2,7 \\
\hline Motcrista & 8 & 2 & 1,8 & - & - & 2 & 1,8 \\
\hline \multirow[t]{2}{*}{ Mecanico } & A & 3 & 2,7 & - & - & 3 & 2,7 \\
\hline & 8 & - & - & - & - & - & - \\
\hline \multirow{2}{*}{ Lavrador } & A & 3 & 2,7 & - & - & 3 & 2,7 \\
\hline & 8 & - & - & - & - & - & - \\
\hline \multirow{2}{*}{ Técnicos } & A & 2 & 1,8 & - & - & 2 & 1,8 \\
\hline & 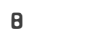 & 2 & 1,8 & - & - & 2 & 1,8 \\
\hline \multirow{2}{*}{ Bancário } & A & 2 & 1,8 & - & - & 2 & 1,8 \\
\hline & $\mathbf{B}$ & - & - & - & - & - & - \\
\hline \multirow[t]{2}{*}{ Industriário } & A & 2 & 1,8 & 2 & 1,8 & 4 & 3,6 \\
\hline & $\theta$ & 3 & 2,7 & 2 & 1,8 & 5 & 0,5 \\
\hline \multirow[t]{2}{*}{ Militar } & A & 2 & 1,8 & - & - & 2 & 1,8 \\
\hline & $\mathbf{B}$ & - & - & - & - & - & - \\
\hline \multirow{2}{*}{$\begin{array}{l}\text { Economista Enge- } \\
\text { nheiros }\end{array}$} & A & 2 & 1,8 & - & - & 2 & 1,8 \\
\hline & 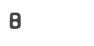 & - & - & - & - & - & - \\
\hline \multirow{2}{*}{$\begin{array}{c}\text { Dentista farmacêu } \\
\text { tico }\end{array}$} & A & 2 & 1,8 & - & - & 2 & 1,8 \\
\hline & $\mathbf{B}$ & - & - & - & - & - & - \\
\hline \multirow[t]{2}{*}{ Estudantes } & A & 1 & 0,9 & 1 & 0,9 & 2 & 1,8 \\
\hline & $\theta$ & 1 & 0,9 & 1 & 0,9 & 2 & 1,8 \\
\hline \multirow{2}{*}{$\begin{array}{l}\text { Mão de obra não } \\
\text { especializada }\end{array}$} & 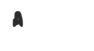 & 1 & 0,9 & 2 & 1,8 & 3 & 2,7 \\
\hline & B & 7 & 6,3 & - & - & 7 & 6,3 \\
\hline TOTAL & & 67 & 60,3 & $\Delta 4$ & 39,6 & 111 & 100,0 \\
\hline
\end{tabular}

* Foram considerados neste item descarregador, ensacador, auxiliar de limpeza e outros semelhantes. 
CIOSAK, S.I. e Colaboradoras - Estudo Preliminar sobre a Influência da Orientaçāo Sistematizada ao Paciente que será Submetido ao Estudo Hemodinâmico. Rev. Bras. Enf.; DF, 35 : 17-38, 1982.

TABELA 3

RELAÇĀO ENTRE SEXO E IDADE DOS PACIENTES DO GRUPO "A"

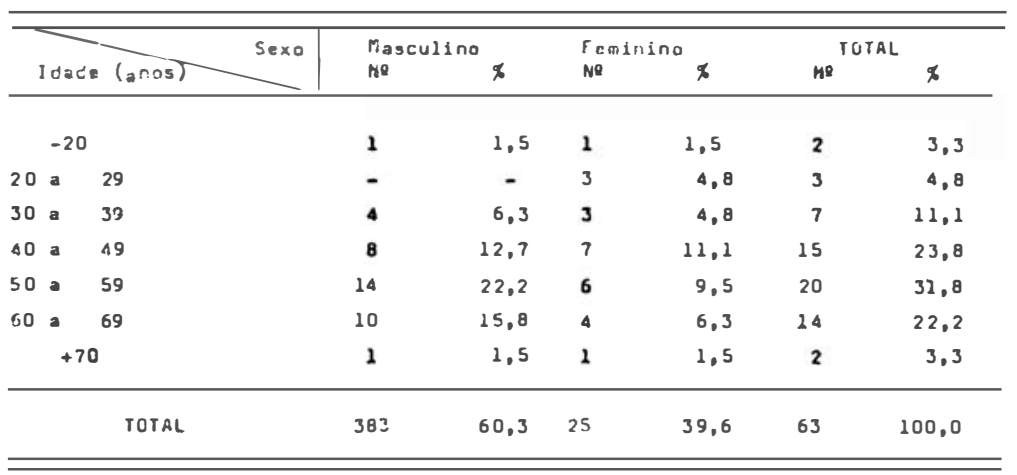

TABELA 4

RELAÇĀO ENTRE SEXO E IDADE DOS PACIENTES DO GRUPO "B”.

\begin{tabular}{|c|c|c|c|c|c|c|c|}
\hline & \multirow{2}{*}{ Sexo } & \multicolumn{2}{|c|}{ Masculino } & \multicolumn{2}{|c|}{ Faninino } & \multicolumn{2}{|c|}{ TOTAL } \\
\hline & & Ne & $x$ & Ne & $x$ & N & $x$ \\
\hline & -20 & 1 & 2,1 & 1 & 2,1 & 2 & 4,2 \\
\hline 20 & a 29 & - & - & 1 & 2,1 & 1 & 2,1 \\
\hline 30 & - 39 & 4 & 8,3 & 2 & 4,2 & 6 & 12,5 \\
\hline 40 & - 49 & 10 & 20,8 & 8 & 16,6 & 18 & 37.5 \\
\hline so & - 59 & 10 & 20,8 & 3 & 6,2 & 13 & 27.1 \\
\hline \multirow[t]{3}{*}{60} & a 69 & 3 & 6,2 & 2 & 4,2 & 5 & 10.4 \\
\hline & +70 & 1 & 2,1 & 2 & 4,2 & 3 & 6,2 \\
\hline & TOTAL & 29 & 60,4 & 19 & 39,5 & 48 & 100,0 \\
\hline
\end{tabular}

TABELA 5

PROCEDENCIA DOS PACIENTES DO GRUPO “A”, SUBMETIDOS AO ESTUDO HEMODINĀMICO EM RELAÇĀO AO SEXO.

\begin{tabular}{|c|c|c|c|c|c|c|}
\hline \multirow{2}{*}{ Procedencia Sexo } & \multicolumn{2}{|c|}{ Masculino } & \multicolumn{2}{|c|}{ Feminino } & \multicolumn{2}{|c|}{ TOTAL } \\
\hline & Ne & 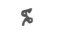 & Ne & $x$ & Ne & $\$$ \\
\hline SR̃O PAUCO - Capital & 8 & 12,7 & 9 & 14,3 & 17 & 26,9 \\
\hline ST̃O PAULO - Interior & 11 & 17,5 & 7 & 11,1 & 18 & 28,6 \\
\hline OUTROS ESTADOS & 19 & 30,2 & 9 & 14,3 & 28 & 44,4 \\
\hline TOTAL & 38 & 60,3 & 25 & 39,6 & 63 & 100,0 \\
\hline
\end{tabular}


CIOSAK, S.I. e Colaboradoras - Estudo Preliminar sobre a Influência da Orientação Sistematizada ao Paciente que será Submetido ao Estudo Hemodinâmico. Rev. Bras. Enf.; DF, 35 : 17-38, 1982.

\section{TABELA 6}

PROCEDENCIA DOS PACIENTES DO GRUPO “B”, SUBMETIDOS AO ESTUDO HEMODINÂMICO EM RELAÇÃO AO SEXO.

\begin{tabular}{|c|c|c|c|c|c|c|}
\hline \multirow{2}{*}{ Procedêncía Sexo } & \multicolumn{2}{|c|}{ Masculino } & \multicolumn{2}{|c|}{ Feminino } & \multicolumn{2}{|c|}{ TOTAL } \\
\hline & Ne & 5 & NQ & $x$ & Ne & $x$ \\
\hline SR̃O pAULO - Capital & 6 & 12,5 & 2 & 4,2 & 8 & 16,6 \\
\hline 5R̃o paulo - Interfor & 7 & 14,6 & 8 & 16,6 & 15 & 31,2 \\
\hline DUTHOS ESTADOS & 16 & 33,3 & 9 & 18,7 & 25 & 52,1 \\
\hline TOTAL & 29 & 60,4 & 19 & 39,5 & 48 & 100,0 \\
\hline
\end{tabular}

Com relação à tabela 7 , verifica-se que a maioria dos pacientes do grupo $\mathrm{A}$ não haviam sido submetidos a este exame $(74,6 \%)$, sendo que $52,4 \%$ dos pacientes não tiveram orientação anterior.
Observa-se ainda que a maioria dos pacientes $(74,6 \%)$, apesar de conhecerem pessoas que já fizeram o estudo hemodinâmico, não receberam orientação anterior.

TABELA 7 EXPERIENCIAS ANTERIORES DOS PACIENTES DO GRUPO "A",
SUBMETIDOS AO ESTUDO HEMODINAMICO.

\begin{tabular}{|c|c|c|c|c|c|c|}
\hline \multirow{2}{*}{$\begin{array}{l}\text { Exp. Alternativa } \\
\text { Anteriores }\end{array}$} & \multicolumn{2}{|c|}{$\sin$} & \multicolumn{2}{|c|}{ NAั0 } & \multicolumn{2}{|c|}{ TOTAL } \\
\hline & ne & $x$ & Ne & $x$ & Ne & 8 \\
\hline Exames anteriores & 16 & 25,4 & 47 & 74,6 & 63 & 100 \\
\hline orientação enterior & 30 & 47,6 & 33 & 52,4 & 63 & 100 \\
\hline $\begin{array}{l}\text { Conheceu alquém que já } \\
\text { fez o exame }\end{array}$ & 47 & 74,6 & 16 & 25,4 & 63 & 100 \\
\hline
\end{tabular}

$\mathrm{Na}$ tabela 8, observa-se que dos $28,0 \%$ pacientes que tinham exames anteriores acharam-no normal e sentiramse bem durante o mesmo; porém agrupados em termos de experiências positivas ("exame bom", "não sentiu nada") e negativas ("horrivel", "mau", "sentiu apavorada", "exame difícil" etc.), verifica-se maior incidência desta última $(60,0 \%)$.
Os pacientes com orientaçōes anteriores, tabela 9, receberam-na através de médicos $(63,4 \%)$, das mais variadas formas. Já o número de orientações dadas por enfermeiras foi muito reduzido, $14,6 \%$, o que demonstra a pouca participação deste profissional neste tipo de atividade, apesar de cientes desta necessidade como foi demonstrada em vários trabalhos neste sentido. HORTA 2 e KAMIYAMA 3,4 . 
CIOSAK, S.I. e Colaboradoras - Estudo Preliminar sobre a Influência da Orientação Sistematizada ao Paciente que será Submetido ao Estudo Hemodinâmico. Rev. Bras. Enf.; DF, 35 : 17-38, 1982.

TABELA 8

SENSAÇÕES RELATADAS PELOS PACIENTES DO GRUPO "A", QUE FIZERAM EXAME ANTERIORMENTE.

\begin{tabular}{|c|c|c|c|}
\hline \multirow{2}{*}{$\begin{array}{c}\text { TIPO OE } \\
\text { EXPERIENCIAS }\end{array}$} & \multirow{2}{*}{ SENSAÇOES } & \multicolumn{2}{|c|}{ FREQUENCIA } \\
\hline & & Ne & $\$$ \\
\hline Experiencias & Normal, bent & 7 & 28,0 \\
\hline Positivas & Não sentiu nada & 4 & 16,0 \\
\hline Expertincias & Doloroso, quase morri & 2 & 8,0 \\
\hline Negativas & Horrível, mau & 5 & 20,0 \\
\hline & Difícil, demorado & 5 & 20,0 \\
\hline & Nervoso, apavorado & 3 & 12,0 \\
\hline TOTAL & & 25 & 100,0 \\
\hline
\end{tabular}

TABELA 9

TIPO DE ORIENTAÇÕES DADAS, RELACIONADAS COM O ORIENTADOR.

\begin{tabular}{|c|c|c|c|c|c|c|c|c|c|c|c|c|}
\hline \multirow[b]{2}{*}{ Orientação dada } & \multicolumn{2}{|c|}{ Miédico } & \multicolumn{2}{|c|}{$\begin{array}{l}\text { outros } \\
\text { pacientes }\end{array}$} & \multicolumn{2}{|c|}{ Colega } & \multicolumn{2}{|c|}{$\begin{array}{l}\text { Enfer- } \\
\text { geiso }\end{array}$} & \multicolumn{2}{|c|}{ famflia } & \multicolumn{2}{|c|}{ TOTAL } \\
\hline & Ne & $x$ & No & $\$$ & NS & $x$ & NE & $x$ & ne & $x$ & No & $x$ \\
\hline $\begin{array}{l}\text { Disseca veia, coloca } \\
\text { sonda no braço e dá } \\
\text { anestesia }\end{array}$ & 13 & 31,7 & 1 & 2,4 & - & - & 2 & 4,8 & - & - & 16 & 39,1 \\
\hline $\begin{array}{l}\text { Filmagem- fotografia } \\
\text { das veias }\end{array}$ & 2 & 4,8 & 1 & 2,4 & - & - & - & - & - & - & 3 & 7,3 \\
\hline $\begin{array}{l}\text { Aconselinou que não } \\
\dot{e} \text { bow }\end{array}$ & 2 & 4,8 & 1 & 2.4 & - & - & - & - & - & - & 3 & 7,3 \\
\hline $\begin{array}{l}\text { Pesquisar jrea que } \\
\text { näo vem sangue }\end{array}$ & 5 & 12,1 & - & - & - & - & 1 & 2,4 & - & - & 6 & 14,6 \\
\hline Simples - bom & 2 & 4,8 & - & - & 1 & 2.4 & - & - & 2 & 4,8 & 5 & 12,2 \\
\hline $\begin{array}{l}\text { Queima tudo por } \\
\text { dentro }\end{array}$ & 1 & 2.4 & 1 & 2,4 & 1 & 2,4 & 2 & 4,8 & - & - & 5 & 12,2 \\
\hline $\begin{array}{l}\text { Não pode dobrar o } \\
\text { brago por } 2 \text { horas }\end{array}$ & 1 & 2.4 & - & - & - & - & - & - & - & - & 1 & 2,4 \\
\hline Não é doloroso & - & - & 1 & 2.4 & - & - & 1 & 2,4 & - & - & 2 & 4,8 \\
\hline TOTAL & 26 & 63.4 & 5 & 12.1 & 2 & 4.8 & 6 & 14,6 & 2 & 4.8 & 41 & 100,0 \\
\hline
\end{tabular}

Através da tabela 10 , observa-se que as informações anteriores dos pacientes do grupo A são bastante variáveis, $29,1 \%$ informaram que eram um exame simples, fácil e tranqüilo; $18,2 \%$ não tinham nada a relatar, mas acharam-no sem riscos; $30,9 \%$ não gostaram e acha- ram o exame horrivel; $20,0 \%$ relatam experiências anteriores como "choque elétrico", "corta o braço", "deita numa coisa". Neste item, alguns pacientes deram mais de uma informação. Num cômputo geral sobressaem as informações negativas. 
CIOSAK, S.I. e Colaboradoras - Estudo Preliminar sobre a Influência da Orientacāo Sistematizada ao Paciente que será Submetido ao Estudo Hemodinâmico. Rev. Bras. Enf.; DF, 35 : 17-38, 1982.

TABELA 10

INFORMAC̣ÕES ANTERIORES DOS PACIENTES DO GRUPO "A" SOBRE O EXAME.

\begin{tabular}{|c|c|c|}
\hline \multirow[t]{2}{*}{ INFORMAGUES ANTERIORES } & \multicolumn{2}{|c|}{ FREQUENCIA } \\
\hline & ne & $\approx$ \\
\hline - Costou, roi bom, simples, fácil, tranquilo & 16 & 29,1 \\
\hline - Nada a relatar, sem risco, não é nada de mais & 10 & 18,2 \\
\hline $\begin{array}{l}\text { - Horrível, dá um calorão, ruim, não gostou, } \\
\text { doi un pouco }\end{array}$ & 17 & 30.9 \\
\hline $\begin{array}{l}\text { - Sente choque elétrico na introdução do catéter, } \\
\text { coloca borracha na veía, corta o braço, deita } \\
\text { numa coixa. }\end{array}$ & 11 & 20,0 \\
\hline $\begin{array}{l}\text { - O coração aparece na TV, porque a cämara entra } \\
\text { na gente }\end{array}$ & 1 & 1,8 \\
\hline$T O Y A L$ & 55 & 100,0 \\
\hline
\end{tabular}

Na tabela 11, verifica-se que a maioria dos pacientes têm uma vaga noção do por quê se faz esse exame, $20,6 \%$ declararam que é para localizar o entupimento da artéria/veia e $36,5 \%$ referem que é para ver o coração; $19,1 \%$ dos pacientes acharam que ele determina o diagnóstico da doença.

TABELA 11

\section{ENTENDIMENTO DOS PACIENTES DO GRUPO "A" SOBRE \\ O ESTUDO HEMODINÂMICO.}

\begin{tabular}{|c|c|c|}
\hline JUSTIFICATIVA SEGUNDO OS PACIENTES & Ne & x \\
\hline - Para ver o coração. & 23 & 36,5 \\
\hline - Localizar o entupimento da artéria/uría & 13 & 20,6 \\
\hline - Determinar o diagnóstico da doença & 12 & 19,1 \\
\hline - Orientar o médico sobre nosso funcionamento & 5 & 7,9 \\
\hline - ผão sabe & 5 & 7,9 \\
\hline - rilmagens das velas do coracão & 4 & 6,3 \\
\hline - Procurar o foco da doenca & 3 & 4,7 \\
\hline - Substitulr a cirurgia & 3 & 4,7 \\
\hline - Para desentup1r a vela e passar a dor & 3 & 4,7 \\
\hline TOTA L & $\underline{6} 3$ & 100,0 \\
\hline
\end{tabular}

Através da tabela 12, constata-se que $73,0 \%$ dos pacientes desconhecem como é feito este exame, do restante, $17,4 \%$ informaram que "corta uma veia e coloca uma sonda". Essas informações, como se pode notar, são vagas e podem criar expectativas negativas ao paciente. 
CIOSAK, S.I. e Colaboradoras - Estudo Preliminar sobre a Influência da Orientaçāo Sistematizada ao Paciente que será Submetido ao Estudo Hemodinâmico. Rev. Bras. Enf.; DF, 35 : 17-38, 1982.

TABELA 12

ENTENDIMENTO DOS PACIENTES DO GRUPO “A" SOBRE

O ESTUDO HEMODINÅMICO.

\begin{tabular}{|c|c|c|}
\hline \multirow{2}{*}{ REALIZACGRO DO EXAMIE SECUNDO OS PACIENTES } & \multicolumn{2}{|c|}{ FREQUEENCIA } \\
\hline & :18 & 8 \\
\hline - Corta uma vela e coloca uma sonda & 11 & 17,4 \\
\hline - Deitado, corta veia e enfia una injeção & 3 & 4,7 \\
\hline - Enfia aparelino na vela e fotograpa o coração & 2 & 3,2 \\
\hline - Injetando contraste através da sonda & 1 & 1,5 \\
\hline - Nä sabe & 46 & 73,0 \\
\hline TOTAL & 63 & 100,0 \\
\hline
\end{tabular}

Com relação às expectativas sobre o exame, a tabela 13 demonstra que $47,6 \%$ dos pacientes referem que "corre tudo bem" e "não seja difícil"; 17,4\% "que descubra a doença"; $31,6 \%$ "que não te- nha que operar" e "que dê bons resultados". Nota-se com estes dados que parte dos pacientes se preocupam com a realização do exame e o restante com o resultado do mesmo.

TABELA 13

\section{EXPECTATIVAS DOS PACIENTES DO GRUPO “A" SOBRE O ESTUDO HEMODINÂMICO.}

\begin{tabular}{|c|c|c|}
\hline \multirow[t]{2}{*}{ EXPECTATIVAS RELATADAS } & \multicolumn{2}{|c|}{ FREQUENCIA } \\
\hline & Ne & $x$ \\
\hline - Que corra tudo bew e não aeja difícil & 30 & 47,6 \\
\hline - Que deacubra a doença & 11 & 17,4 \\
\hline - Que dé bor resultado & 10 & 15,8 \\
\hline - Que não tenha que operar & 10 & 15,8 \\
\hline - Que desentupa as veias & & 1,5 \\
\hline - Que seja operada, pols näo se sente ber & & 1,5 \\
\hline TOTAL & 63 & 100,0 \\
\hline
\end{tabular}

Quanto às informações que gostariam de receber antes do exame, podese constatar pela tabela 14 que $61,9 \%$ dos pacientes gostariam de saber como é feito; já $14,3 \%$ não gostariam de saber nada sobre o exame, alegando ficarem mais ansiosos e amedrontados. 
CIOSAK, S.I. e Colaboradoras - Estudo Preliminar sobre a Influência da Orientação Sistematizada ao Paciente que será Submetido ao Estudo Hemodinâmico. Rev. Bras. Enf.; DF, 35 : 17-38, 1982.

TABELA 14

INFORMAÇŌES QUE OS PACIENTES DO GRUPO “A” GOSTARIAM DE RECEBER ANTES DO ESTUDO HEMODINÂMICO.

\begin{tabular}{|c|c|c|}
\hline INFORMACGES DESEJADAS & \multicolumn{2}{|c|}{ FREQUंÜNCIA } \\
\hline - Como é Peito & 39 & 61,9 \\
\hline - Nada & 9 & 14,3 \\
\hline - O resultado & 7 & 11,1 \\
\hline - Existência de riscos & 6 & 9,5 \\
\hline - So precisa permanecer no Hospitel, após o exame & 2 & 3,2 \\
\hline TOTAL & 63 & 100,0 \\
\hline
\end{tabular}

Com relação ao estado emocional no pré-exame, referido pelos pacientes na tabela 15 , verifica-se que $44,4 \%$ referem "nervoso", "medo"; 17,4\% "ansioso", "irrequieto" e "preocupado". Embora
$39,6 \%$ dos pacientes tenham referido "calma e tranqüilidade", observou-se que o seu comportamento irrequieto durante a aplicação do formulário não condizia com a resposta dada.

TABELA 15

ESTADO EMOCIONAL DOS PACIENTES DO GRUPO "A" NO PRE-EXAME.

\begin{tabular}{|c|c|c|}
\hline ESTADO EMOCIONAL REFERIDO & Ne & $x$ \\
\hline - Nervoso, medo & 28 & 44,4 \\
\hline - Calmo, bew, tranquilo & 25 & 39,6 \\
\hline - Ansioso, irriquieto, preocupado & 10 & 17.4 \\
\hline TOTA & 63 & 100,0 \\
\hline
\end{tabular}

As tabelas de 16 a 23 referemse aos pacientes do grupo $\mathbf{A}$, após a realização do exame.

Quanto aos sentimentos referidos pelos pacientes do grupo A em relação ao exame, após realizá-lo, verifica-se pela tabela 16 que $73,0 \%$ dos pacientes consideraram-no "bom e fácil", seguido de $9,5 \%$ que o classificaram como "ótimo". Apenas 4,7\% classificaram-no como horrivel, o que coincidiu com as intercorrências durante a realização do exame (tabela 17).

Nota-se ainda, pela tabela seguinte (tabela 17), que a incidência de intercorrências, durante o exame, é baixa, perfazendo um total de $4,5 \%$ no grupo $\mathrm{A}$ e $6,8 \%$ no grupo $B$, apesar de que, segundo informações do paciente, provoca traumas emocionais duradouro. 
CIOSAK, S.I. e Colaboradoras - Estudo Preliminar sobre a Influência da Orientação Sistematizada ao Paciente que será Submetido ao Estudo Hemodinâmico. Rev. Bras. Enf.; DF, 35 : 17-38, 1982.

TABELA 16

SENTIMENTOS EM RELAC̣ĀO AO EXAME, REFERIDOS PELOS PACIENTES DO GRUPO "A".

\begin{tabular}{|c|c|c|}
\hline SENTIMENTOS REFERIOOS & 0 & 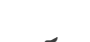 \\
\hline - Bom - fácil & 46 & 73,0 \\
\hline$-\sigma t 1 m 0$ & 6 & 9,5 \\
\hline - Não gostou & 4 & 6,3 \\
\hline - Tolerável & 4 & 6,3 \\
\hline - Horrível & 3 & 4,7 \\
\hline TOTAL & 63 & 100,0 \\
\hline
\end{tabular}

TABELA 17

INTERCORRENCIAS OCORRIDAS DURANTE A REALIZAÇAO DO EXAME DOS GRUPOS "A" E "B".

\begin{tabular}{|c|c|c|c|c|}
\hline \multirow{2}{*}{ INTERCOARENCIAS } & \multicolumn{4}{|c|}{ FREOUENCIA } \\
\hline & GR & $\hat{x}$ & $\begin{array}{l}\text { GRUPO } \\
\text { Ne }\end{array}$ & 8 \\
\hline = Nennuma & 60 & 95,2 & 45 & 93.7 \\
\hline $\begin{array}{l}\text { - Oificuldade encontrar veia, com reali- } \\
\text { zeção do exame nos } 2 \text { membros superiores }\end{array}$ & 1 & 1,5 & 2 & 4,7 \\
\hline - Oissecção acidental de artéria & 1 & 1,5 & - & - \\
\hline - Complementeção Imediata do examz & 1 & 1,5 & - & - \\
\hline - Hipotensão e bradicarodia & - & - & 1 & 2,1 \\
\hline TOTAL & 63 & 100,0 & 48 & 100,0 \\
\hline
\end{tabular}

Através da tabela 18, observa-se que $41,4 \%$ classificaram a orientação como boa; $28,5 \%$ como ótima. Como não houve nenhuma opinião invalidando esta orientação, acredita-se que os $14,3 \%$ dos pacientes, que na tabela 15 referiram não quererem nenhuma informação, fizeram-no por falta de esclarecimento, discernimento ou até decorrente de sua própria condição de escolaridade. 
CIOSAK, S.I. e Colaboradoras - Estudo Preliminar sobre a Influência da Orientação Sistematizada ao Paciente que será Submetido ao Estudo Hemodinâmico. Rev. Bras. Enf.; DF, 35 : 17-38, 1982.

TABELA 18

OPINIĀO SOBRE A ORIENTAÇĀO DADA, REFERIDA PELOS PACIENTES DO GRUPO "A".

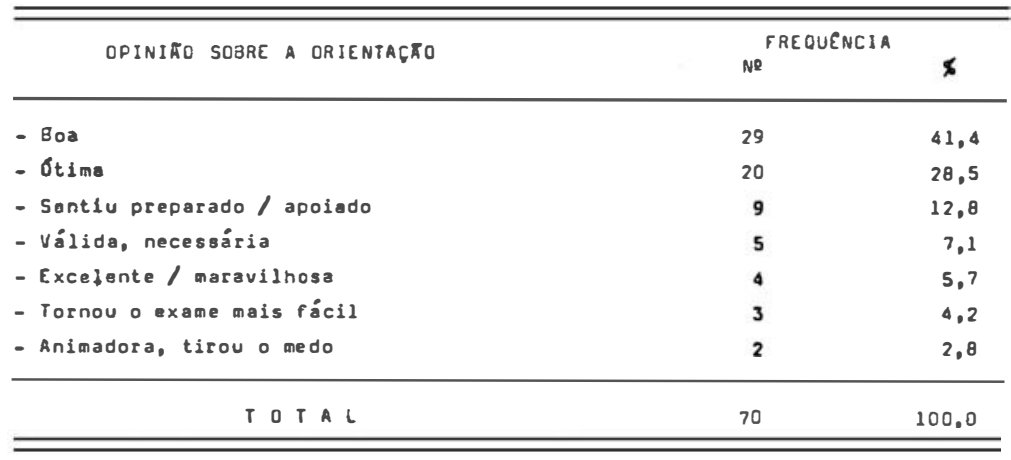

Nota: Alguns pacientes deram mais de uma opinião.

Os sentimentos dos pacientes do grupo A, relacionados à orientação dada, após a realização do estudo hemodinâmico, podem ser observados na tabela 19 , onde se verifica que $43,8 \%$ dos pacientes afirmam que a orientação deixa-os preparados para o que irá acontecer; $36,9 \%$ disseram-se calmos e tranqüilos. Apenas 2,7\% não responderam a esta pergunta.

Os resultados das tabelas 17 e 18 mostram que o homem, quando se encontra em ambiente não familiar, sobretudo em meio desconhecido, incon- trolável e onde não sabe o que dele se espera, sente muita carência de segurança e apoio (MASLOW ${ }^{5}$ ), dai $\mathrm{a}$ importância do enfermeiro desempenhar funçōes que reduzam tensōes e promovam segurança ao paciente.

$\mathrm{Na}$ tabela 20, relacionam-se respostas sobre a suficiência das informaçōes e verifica-se que $94,0 \%$ dos pacientes consideram que nada faltou à orientação dada, as outras respostas não foram significativas pela incidência e pelo seu con'teúdo.

TABELA 19

SENTIMENTOS DOS PACIENTES DO GRUPO "A", APÓS A REALIZAÇÃO

DO ESTUDO HEMODINAMICO, COM RELAÇĀO A ORIENTAÇĀO DADA.

\begin{tabular}{|c|c|c|}
\hline \multirow[b]{2}{*}{ SENTIMENTOS REFERIOCS } & \multicolumn{2}{|c|}{ FREQUENCIA } \\
\hline & Ne & 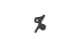 \\
\hline - Deixa-preparado para o que val acontecer & 32 & 43,8 \\
\hline - Sentiu-se calmo, tranquilo & 27 & 36,9 \\
\hline - Oar corager, apoio & 7 & 9,5 \\
\hline - racllita o exame, maior colaboração & 3 & 4.1 \\
\hline - Recordou tudo que sente & 2 & 2,7 \\
\hline - Nāo respondeu & 2 & 2,7 \\
\hline$T O T A L$ & 73 & 100,0 \\
\hline
\end{tabular}


CIOSAK, S.I. e Colaboradoras - Estudo Preliminar sobre a Influência da Orientação Sistematizada ao Paciente que será Submetido ao Estudo Hemodinâmico. Rev. Bras. Enf.; DF, 35 : 17-38, 1982.

TABELA 20

NECESSIDADE DE COMPLEMENTAÇĀO DA ORIENTAÇĀO DADA, SEGUNDO PACIENTES DO GRUPO "A".

\begin{tabular}{|c|c|c|}
\hline COMPLEMENTAÇFO SOLICITADA & \multicolumn{2}{|c|}{ FREQUENCIA } \\
\hline - Nada & 59 & 94,0 \\
\hline - Não sabia do calor & 1 & 1,5 \\
\hline - Dizer que podia tirar cópia do exame & 1 & 1,5 \\
\hline - Efeitos colaterais & 1 & 1,5 \\
\hline - Não respondeu & 1 & 1,5 \\
\hline$T O T A L$ & 63 & 100,0 \\
\hline
\end{tabular}

Quanto às modificações do conhecimento anterior ocorridas, verifica-se que dos $39,6 \%$ dos pacientes, que já tinham experiências anteriores, $15,8 \%$ consideraram-no mais rápido e mais fácil; $11,1 \%$ referiram ter mais coragem e melhor orientação em relação ao exame (tabela 21).

Em relação às sugestões dadas pelos pacientes, para melhorar $\mathrm{Q}$ atendimento durante a realização dos exames, $79,3 \%$ nada sugeriram, ou melhor, expressaram satisfação com o atendimento, como se pode observar pela tabela 22; onde $57,1 \%$ dos pacientes nada acrescentaram, $11,1 \%$ acharam tudo ótimo e $11,1 \%$ consideraram o atendimento completo. Entretanto, $15,8 \%$ solicitam diminuir o tempo de espera, antes da realização do exame, o que leva a aumentar o nível de sua ansiedade, porém nem sempre é possível minimizar esta espera, devida às intercorrências que podem ocorrer devida à suspeita diagnóstica ou gravidade dos pacientes.

TABELA 21

MODIFICAÇÕES DO CONHECIMENTO ANTERIOR, REFERIDAS PELOS PACIENTES DO GRUPO "A".

\begin{tabular}{|c|c|c|}
\hline MODIFICACGES DO CONHECIMENTO ANTERIOR & \multicolumn{2}{|c|}{ FREQUENCIA } \\
\hline - Desconhecia o exame & 38 & 60,3 \\
\hline - Foi mais fácil, rápido & 10 & 15,8 \\
\hline $\begin{array}{l}\text { - Fol bem orientado, teve mals coragem } \\
\text { melhor atendimento }\end{array}$ & 7 & 11,1 \\
\hline - Demorou, sentiu mais calor & 3 & 4,7 \\
\hline - Nenhuma & 3 & 4,7 \\
\hline - Não gostou de repetír, desconhece a razão & 1 & 1,5 \\
\hline - Melhor do que contaram & 1 & 1,5 \\
\hline$T O T \wedge L$ & 63 & 100,0 \\
\hline
\end{tabular}


CIOSAK, S.I. e Colaboradoras - Estudo Preliminar sobre a Influência da Orientação Sistematizada ao Paciente que será Submetido ao Estudo Hemodinâmico. Rev. Bras. Enf.; DF, 35 : 17-38, 1982.

TABELA 22

SUGESTÕES PARA MELHORAR O ATENDIMENTO, REFERIDO PELOS

PACIENTES DO GRUPO "A", NO PÓS-CATETERISMO.

\begin{tabular}{lcc}
\hline \hline SUGESTOES DADAS & \multicolumn{2}{c}{ FREQUENCIR } \\
\hline - Nada mais * & 36 & 57,1 \\
- Olminuir o tempo de espera & 10 & 15,8 \\
- Tudo ótimo * & 7 & 11,1 \\
- Foi complete * & 7 & 11,1 \\
- Internar entes & 1 & 1,5 \\
- Explicacão sobre intercorrências & 1 & 1,5 \\
- Não lembra & 1 & 1,5 \\
\hline & 63 & 100,0 \\
\hline
\end{tabular}

* Consideramos estes resultados como satisfação ao atendimento prestado, que agrupades correspondem a 50 respostas, $79,3 \%$.

$\mathrm{Na}$ tabela 23 , verifica-se que $60,3 \%$ dos pacientes resrionderam sim, sem hesitar, à necessidade de repetir o exame, já $25,4 \%$ repetiriam-no apenas se ${ }^{-}$fosse necessário.

A partir da tabela 24, procura-se relacionar os sentimentos dos pacientes do grupo $\mathrm{B}$, e com relação ao exame realizado, verifica-se pela tabela 24 que
$43,8 \%$ desses pacientes acharam o exame suportável e ficaram nervosos e $20,8 \%$ acharam muito bom. Observa-se que neste grupo, de não orientados, foi maior a incidência de sen'timentos negativos que a do grupo $\mathrm{A}$, alertando a enfermagem quanto ao valor do aspecto emocional durante a realização do exame.

TABELA 23

RESPOSTA SGBRE A POSSIBILIDADE DE REALIZAÇĀO DE NOVO EXAME; SEGUNDO PACIENTES DO GRUPO "A".

\begin{tabular}{|c|c|c|}
\hline \multirow{2}{*}{ RESPOSTAS OBTIDAS } & \multicolumn{2}{|c|}{ FREQUE NC IA } \\
\hline & Ne & $x$ \\
\hline$-\sin$ & 38 & 60,3 \\
\hline - Se preciso & 16 & 25,4 \\
\hline - Faria con mado & 3 & 4,7 \\
\hline - Acho que não & 2 & 3,2 \\
\hline - Munca & 1 & 1,5 \\
\hline - Só en caso de vida ou morte & 1 & 1,5 \\
\hline - Só com anestesía geral & 1 & 1,8 \\
\hline - Com mais confianga & 1 & 1,5 \\
\hline TOTAL & 63 & 100,0 \\
\hline
\end{tabular}


CIOSAK, S.I. e Colaboradoras - Estudo Preliminar sobre a Influência da Orientação Sistematizada ao Paciente que será Submetido ao Estudo Hemodinâmico. Rev. Bras. Enf.; DF, 35 : 17-38, 1982.

TABELA 24

SENTIMENTOS SOBRE O EXAME RELATADOS PELOS PACIENTES DO GRUPO "B".

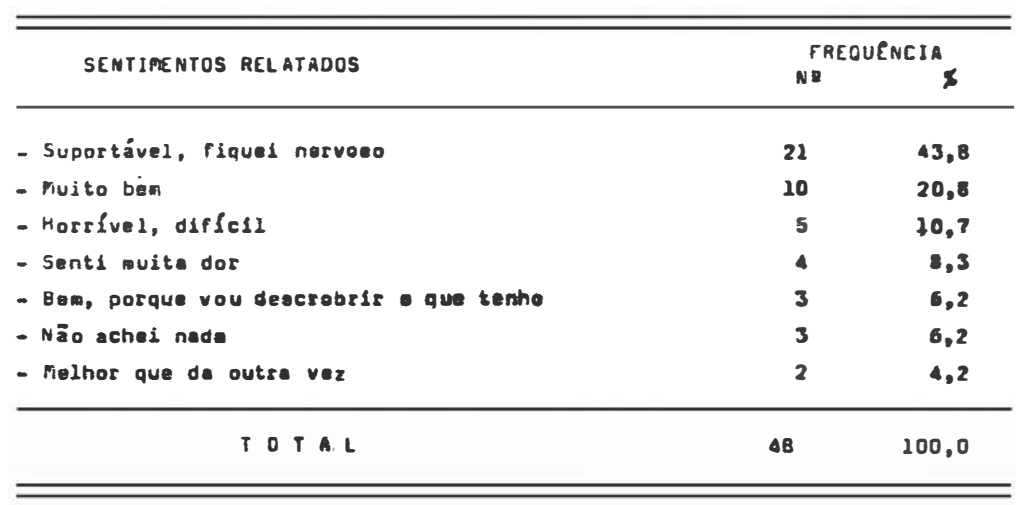

Pela tabela 25 , observa-se que $41,6 \%$ dos pacientes possuiam conhecimentos anteriores sobre o exame e $58,3 \%$ desconheciam-no, e relacionando com a tabela seguinte (tabela 26), verifica-se que $70,8 \%$ dos pacientes sen'tiram necessidade de orientação prévia, por esta pcrcentagem, conclui-se que alguns dos pacientes que já tinham conhecimento anterior, sentiram esta mesma necessidade. Nesta mesma tabela, 27,1\% dos pacientes responderam negativam ənte a essa necessidade, atribui-se isto à falta de esclarecimentos sobre o exame, visto que alguns deles não sabiam nem mesmo o nome do exame.

TABELA 25

CONHECIMENTO ANTERIOR DO GRUPO "B" SOBRE O EXAME.

\begin{tabular}{ccc}
\hline \hline \multirow{2}{*}{ CONHECIMENTO } & \multicolumn{2}{c}{ FREQUENCIA } \\
& NQ & $\%$ \\
\hline SIM & 20 & 41,6 \\
NAO & 28 & 58,3 \\
\hline TOTAL & 48 & 100,0 \\
\hline \hline
\end{tabular}


CIOSAK, S.I. e Colaboradoras - Estudo Preliminar sobre a Influência da Orientação Sistematizada ao Paciente que será Submetido ao Estudo Hemodinâmico. Rev. Bras. Enf.; DF, 35 : 17-38, 1982.

TABELA 26

NECESSIDADE DE ORIENTAÇĀO REFERIDA PELO GRUPO "B".

\begin{tabular}{llcc}
\hline \hline \multirow{2}{*}{ NECESSIDADE DE ORIENTAÇAO } & NE & FREQUENCIA \\
& & 34 & 70,8 \\
SIM & 13 & 27,1 \\
NATO & 1 & 2,1 \\
NATO SABE & 48 & 100,0 \\
\hline & & & \\
\hline \hline
\end{tabular}

TABELA 27

OPINIĀO DOS PACIENTES DO GRUPO "B" SOBRE A NECESSIDADE DE ORIENTAC̄ĀO.

\begin{tabular}{|c|c|c|}
\hline \multirow{2}{*}{ OPINIOES } & \multicolumn{2}{|c|}{ FBEQUENCIA } \\
\hline & Ne & $\%$ \\
\hline - Sabe-se tudo o que vai acontecer & 29 & 56,8 \\
\hline - Não, deixa a pessoa mais nervosa & 7 & 13,7 \\
\hline - Acaba com o medo & 5 & 9,8 \\
\hline - Deixa mais calmo & : & $5, \theta$ \\
\hline - Não, porque já fez exame & 3 & $5, \theta$ \\
\hline - E ideal para quem nunca $f a z$ & 3 & 5,8 \\
\hline - Não sabe & 1 & 1,9 \\
\hline T O TA L & $51 *$ & 100,0 \\
\hline
\end{tabular}

$\mathrm{Na}$ tabela 27, procura-se saber a opiniāo dos pacientes sobre a necessidade de uma orientação prévia e constata-se que $56,8 \%$ dos pacientes gostariam de ser orientados para conhecer a experiência que irão passar e, com isso, adquirir maior segurança, ficarem calmos e diminuir o medo. Os pacientes que já fizeram o exame, recomendamno para os que não o fizeram. Alguns asharam desnecessária a orientação, alegando que ficariam nervosos $(13,7 \%)$; observcu-se que a ansiedade e o desconhecimento de determinados pacientes os levam à rejeição da orientação prévia.

* Algumas pessoas apresentaram mais de uma opinião. 
CIOSAK, S.I. e Colaboradoras - Estudo Preliminar sobre a Influência da Orientação Sistematizada ao Paciente que será Submetido ao Estudo Hemodinâmico. Rev. Bras. Enf.; DF, 35 : 17-38, 1982.

TABELA 28

MODIFICAÇÕES SENTIDAS PELOS PACIENTES DO GRUPO "B", EM RELAÇÃO AO CONHECIMENTO ANTERIOR SOBRE O EXAME.

\begin{tabular}{|c|c|c|}
\hline \multirow{2}{*}{ MOOIFICAÇOES SENTIOAS } & \multicolumn{2}{|c|}{ FREQUENCIA } \\
\hline & NQ & $\varnothing$ \\
\hline - Não conhecia nada & 30 & 62,5 \\
\hline - Fol melhor que o outro & 6 & 12,5 \\
\hline - Pensou que fosse mais fácil & 5 & 10,4 \\
\hline - Sentiu mais dor que o outro & 3 & 6,3 \\
\hline - Não houve modificação & 2 & 4,2 \\
\hline - Foi o que esoerava & 1 & 2,1 \\
\hline - Fol mals calmo & 1 & 2,1 \\
\hline T D T A L & $4 \theta$ & 100,0 \\
\hline
\end{tabular}

Verifica-se pela tabela 28 que $62,5 \%$ dos pacientes não conheciam nada do exame, porém, aqueles que já o tinham feito, $12,5 \%$, acharam-no melhor que o outro e somente para $4,2 \%$ não houve nenhuma modificação.

TABELA 29

SUGESTÕES DOS PACIENTES DO GRUPO “B”, PARA MELHORAR O ATENDIMENTO DURANTE A REALIZAÇÃO DO EXAME.

\begin{tabular}{ccc}
\hline \hline Sugestöes & FREQuENCIA \\
\hline - Preparo prévio & Ne & $\%$ \\
- Manter o atendimento & 26 & 54,1 \\
- Esperar menos tempo & 8 & 16,7 \\
- Nenhuma sugestão & 7 & 14,6 \\
\hline T OTA L & 7 & 14,6 \\
\hline \hline
\end{tabular}

Na tabela 29, selecionaram-se sugestões dos pacientes para melhorar o atendimento durante o exame e verificou-se que $54,1 \%$ dos pacientes sugeriram que tivessem um preparo prévio.
Esse dado é relevante para alertar a enfermagem sobre a real necessidade dos pacientes que, apesar de incoerentes em ou'tras respostas, nesta evidencia-se o valor da orientação prévia. 
CIOSAK, S.I. e Colaboradoras - Estudo Preliminar sobre a Influéncia da Orientação Sistematizada ao Paciente que será Submetido ao Estudo Hemodinâmico. Rev. Bras. Enf.; DF, 35 : 17-38, 1982.

TABELA 30

RESPOSTAS DOS PACIENTES DO GRUPO "B" SOBRE A POSSIBILIDADE DE REALIZAC̣AOO DE NOVO EXAME.

\begin{tabular}{|c|c|c|}
\hline \multirow{2}{*}{ RESPOSTAS DOS PACIENTES } & \multicolumn{2}{|c|}{ FREQUENCIA } \\
\hline & Ne & $x$ \\
\hline - Se for necessário & 25 & 52,0 \\
\hline$-\operatorname{sim}$ & 18 & 37,5 \\
\hline - Năo & 2 & 4,2 \\
\hline - só ex último caso & 2 & 4,2 \\
\hline - Nunca, prefiro morrer & 1 & 2,1 \\
\hline$T O T A L$ & 48 & 100,0 \\
\hline
\end{tabular}

Na tabela 30 , embora $37,5 \%$ dos pacientes tenham dito que repetiriam o exame, constata-se que a experiência foi traumatizante, pois $\mathbf{5 2 , 0 \%}$ mostraram-se relutantes em repetir o exame, propondo-se a tal, apenas, em caso de necessidade.

Comparando-se o aspecto comportamental entre os dois grupcs A e B, cbsarvou-se nos pacientes do grupo $\mathbf{A}$ uma maior colaboração e participação durante a realização do exame, enquanto que os pacientes do grupo B mantiveram-se apreensivos e poucos participantes. Os aspectos analisados permitiram comprovar que o grupo submetido a ação sistematizada da enfermagem voltada para a orientação prévia, reagiram melhor diante das solicitações médicas, mostraram-se mais seguros, colaborando melhor e menos susceptível de alterações físicas e comportamentais, visto que o paciente ansioso e apreensivo tolera pouco a dor e apresenta incidência acentuada de complicações, FONTES ${ }^{1}$.
Evidenciou-se também a importância da enfermagem no que concerne ao aspecto psicológico do paciente, constituindo-se esta em uma das atividades de maior destaque.

\section{CONCLUSÃO}

Do presente estudo, conclui-se que a orientação sistematizada no preparo psicológico do paciente submetido ao estudo hemodinâmico é eficaz, pois, além de promover maior grau de conhecimento do paciente com relação ao exame, promove ajuda na realização do mesmo, pois, através de sentimentos de segurança e diminuição de expectativas, torna-os mais participantes durante a realização do exame e menos susceptíveis de alterações físicas e comportamentais.

Daí a necessidade do enfermeiro d?sempenhar funções para reduzir tensões, mediante promoção de orientação ou relacionamento positivo, que favoreçam sen'timentos de segurança e cooperação. 
CIOSAK, S.I. e Colaboradoras - Estudo Preliminar sobre a Influência da Orientação Sistematizada ao Paciente que será Submetido ao Estudo Hemodinâmico. Rev. Bras. Enf.; DF, 35 : 17-38, 1982.

B I B L I O G R A F I A

1. FONTES, M. C. et alii. O trauma cirúrgico - importância da orientação pré-operatória. Rev. Bras. Enf., 33 (2) :194-200, 1980.

2. HORTA, W. A. Necessidades humanas básicas: consideraçōes gerais. Enf. Nova Dimens., 1 (5) :266-8, 1975.

3. KAMIYAMA, Y. O doente hospitalizado e sua percepcāo quanto a prioridade de seus problemas. São Paulo, 1972. (Tese de Doutoramento - Escola de Enfermagem da Universidade dc São Paulo).

4. Assistência centrada na identidade social, aspectos psicossociais do cuidado de enfermagem ao paciente de hepatite infecciosa. São Paulo, 1979. (Tese de Livre Docência - Escola de Enfermagem da Universidade de São Paulo).

5. MASLOW, A. H. Motivation and personality. 2nd edition, New York, Harper \& Row, 1970, 369 p.

\section{ANEXO}

ORIENTAÇĀO DADA PELA ENFERMEIRA AOS PACIENTES QUE IRĀO SE SUBMETER AO ESTUDO HEMODINÂMICO

"O exame é simples, tem a duração de 30 a 40 minutos.

É feito pelo braço direito, na maioria das vezes, com anestesia local em adultos e geral em crianças.

É Clada uma injeção na priga do cotovelo do braço direito, ou outro local onde será feito o exame, e injetado o anestésico. Quando está sendo injetado anestésico, sente-se um ardor no local.

Após isso, faz-se um pequeno cortc para procurar uma veia ou uma artéria ou os dois, a depender do estudo que será feito. Esta veia fica junto de um nervo, que precisa ser separado, para evitar traumatismos. Quando está sendo feito isso, sente-se uma espécie de "choque" no local, mas de efeito passageiro.

Logo após se injeta uma medicação na veia para não formar coágulos na circulação durante o exame e o que se sente é um calor forte na mão.

É introduzido um cateter (sonda) e inicia-se o exame, injetando-se manualmente contraste nos locais em estudo. Durante, ou no final do exame, é feita uma ou mais injeções de contraste, sob alta pressão, que dará uma sensação de calor bem forte que dura 20 segundos.
Durante todo o exame, o médico avisará o que irão sentir. A colaboração de vocês é de suma importância para $c$ b.m andamento e rapidez do exame. Para isso, ele solicitará:

- tossir, para eliminar mais rápido o contraste injetável;

- respirar fundo, segurar e respirar normal, para maior expansão do tórax e melhor visão do que está sendo feito;

- virar de um lado e do outro, para melhor visualização do coracão.

As reações, que poderão sentir durante o exame e que são consideradas normais, são: náuseas, dor de cabeça, dor no peito (não muito forte), tontura.

Sentindo qualquer uma delas, avisar a pessoa que estiver mais próxima.

Ficarão com você em sala um médico e duas auxiliares de enfremagem.

A sala de exames possui vários aparelhos, com a finalidade de facilitar a realização do exame. São colocadas placas de metal nos braços e pernas, para controle de seu coração, através do ECG, durante toda a realização do exame. 
CIOSAK, S.I. e Colaboradoras - Estudo Preliminar sobre a Influência da Orientação Sistematizada ao Paciente que será Submetido ao Estudo Hemodinâmico. Rev. Bras. Enf.; DF, 35 : 17-38, 1982.

Os aparelhos constam de: mesa giratória, com cabo manual, controlada pelo médico. Esta mesa se move para a frente, para 'trás e para os lados. O outro aparelho (poligrafo) registra o ECG e pressões. Há uma televisão onde se vê tudo o que está sendo feito. Acima de vocês, fica um tubo, que é a máquina filmadora.

Após o exame, deve-se fazer exercícios de abrir e fechar a mão e só dobrar o membro cateterizado após 2 horas.

Os senhores sairão da sala de maca e permanecerão 15 minutos no repouso, para observação de intercorrências.
Após, são encaminhados para o quarto, ainda de maca e receberão alta no outro dia, às nove horas.

O relatório será entregue pela secretaria à tarde, a partir das 17 horas.

\section{BOA SORTE.'}

Obs.: Para pacien'tes com dificuldades de entendimento, ou com: conhecimentos anteriores, foram prestadas informações complementares, a partir da orientação dada. As orientações foram dadas em conjunto na sala de pré-exames. 